\title{
AN EVALUATION OF THE MATHEMATICAL MODELS OF ENERGY PILES
}

\author{
Jakub Oravec ${ }^{1 *}$, Ondřej Šikula ${ }^{1}$, Iva Nováková ${ }^{1}$
}

\begin{abstract}
A current trend is to reduce the energy performance of buildings by using alternative sources for heating and cooling. One of the most promising, and so far unprecedented sources of heating and cooling, is the use of energy from the earth using the thermally-activated foundation piles of a building, the so-called energy piles. The paper deals with an overview and comparison of computer-aided analytical models of energy piles. The individual analytical models are compared (categorized) from the point of view of their physical complexity, computational costs, and thus their usability for the purpose of optimizing energy-pile equipment or assessing the long-term energy efficiency of an energy pile field. Selected mathematical models were algorithmized, and the results obtained were compared with a more robust numerical solution performed using CalA 4 software.
\end{abstract}

\section{Address}

1 Institute of Building Services, VUT Brno, Faculty of Civil Engineering, Veveř́ 331/95, 60200 Brno, Czech Republic

* Corresponding author: jakub.oravec@vutbr.cz

\section{Key words}

- Energypile,

- Analytical models, G-function,

- Infinite line source model,

- Cylindrical source model,

- Finite line source model.

\section{INTRODUCTION}

An energy pile, which is a thermally-activated pile, is a vertical foundation structure in which a ground heat exchanger is placed and by means of which heat is transferred between the soil and the heat transfer medium. The main advantage is the use of geothermal energy, which is one of the renewable sources of heat compared to the frequently-used geothermal borehole. Their use is cost-effective in buildings where pile foundations are necessary. In general, there are two ways of modeling the thermal processes that take place in an energy pile. Using analytical or numerical models. Analytical models contain several simplifications, which significantly reduce their computational complexity. On the other hand, numerical models can simulate the thermal processes taking place in a pile and the surrounding soil quite accurately, but their computational cost significantly increases. Most of the research carried out to date deals with boreholes. Much less research deals with thermally-activated piles. The aim of this paper is to compare selected analytical models originally developed for borehole modeling with a numerical solution and determine whether they can be effectively used for modeling energy piles.

\section{ANALYTICAL MODELS}

The theoretical basis for most conventional analytical models for modeling thermal processes is based on Kelvin's theory of heat sources, the Laplace transform method, and Duhamel's theorem (Ingersoll et al., 2007), (Carslaw et al., 1959). In the analytical calculation of energy piles, heat transfer is generally modeled in 2 areas, i.e., in the soil and in the pile, which are simply considered to be homogeneous mediums. All the models use simplifying assumptions. The most common are:

- the soil is considered to be infinite or semi-infinite

-there is a uniform initial constant temperature

- the boundary condition for the pile or pipe wall is a constant heat flow or a constant temperature

- the soil is considered to have equivalent thermal conductivity

The searched-for quantity for modeling these thermal processes is the heat flux $q[\mathrm{~W} / \mathrm{m}]$ between the heat transfer medium and the soil as a function of time at a certain temperature difference between the heat transfer medium and the soil, which is dependent on the total thermal resistance between them $(\mathrm{R}(\mathrm{t})[\mathrm{mK} / \mathrm{W}])$. 


$$
q=\frac{T_{f}(t)-T_{0}}{R(t)}=\frac{T_{f}(t)-T_{0}}{R_{p}+G(x, t)}
$$

The mean temperature of the heat transfer medium and the temperature of the soil are known quantities. The analytical models deal with finding an equation to determine the total thermal resistance $\mathrm{R}$ (t). The total thermal resistance in the analytical models is divided into 2 components, i.e., time-dependent and time-independent. The time-dependent component is referred to as the G-function - $G(x, t)$, which is the temperature response of the soil with respect to the unit change in the heat flow. The G function describes the thermal processes taking place in the soil outside the piles. The time-independent component of the total thermal resistance $\left(R_{p}\right)$ describes the thermal events taking place in the pile itself and indicates that the non-stationary conduction of heat in the pile is ignored. The time-independent component is therefore the thermal resistance between the circulating heat carrier and the pile's outer wall (Li and Lai, 2015).

\section{THERMAL RESISTANCE OF AN ENERGY PILE}

The basic prerequisite for determining the thermal resistance of the pile $\mathrm{R}_{\mathrm{p}}$ is that the thermal processes in the pile are at a steady state. This state is defined as (rp [m] is the pile radius) (Li and Lai, 2015):

$$
t>5 \cdot\left(r_{p}^{2} / a_{p}\right)
$$

Thus, at time $\mathrm{t}[\mathrm{s}]$, the heat flow in the pile can be considered to be steady, and the temperature difference between the heat carrier and the pile wall is constant. The models for $R_{p}$ and thus for the G-functions are only applicable when this criterion is met. The thermal resistance of the pile $\mathrm{R}_{\mathrm{p}}$ mainly depends on the piping configuration and the thermal properties of the concrete and surrounding soil. This local heating process has three components:

$-\mathrm{R}_{\mathrm{f}}$ is the thermal resistance in the convective heat transfer between the circulating fluid and inner surface of the exchanger tube;

$-\mathrm{R}_{\mathrm{pw}}$ is the thermal resistance of the conduction of the heat transfer through the wall of the exchanger tube;

$-\mathrm{R}_{\mathrm{c}}$ is the thermal resistance of the conduction of the heat transfer through the filler material.

The total thermal resistance can be defined as the sum of these three components. The models for calculating $\mathrm{R}_{\mathrm{p}}$ can be divided into empirical and theoretical models. Empirical models are widely used due to their simplicity and have been used in our comparison. The empirical 1D model used in our evaluation simplifies the actual U-shaped circular duct pipe to one pipe of an equivalent diameter. This simplifies the two-dimensional geometric region to a concentric annular region, thereby simplifying the complicated multi-dimensional problem to a one-dimensional problem (Gu et al., 1998).

$$
\mathrm{R}_{\mathrm{p}}=\frac{1}{2 \pi \lambda_{\mathrm{p}}} \ln \left(\frac{\mathrm{r}_{\mathrm{p}}}{\mathrm{r}_{\mathrm{pipe}}} \sqrt{\frac{\mathrm{r}_{\text {pipe }}}{\mathrm{D}}}\right)
$$

\section{CALCULATING THE G-FUNCTION}

Models for calculating the G-function are based on the theoretical foundations proposed by Ingersoll et al. (2007). In his work he presented the Infinite line source model (IFLSM), which allows the temperature field in the ground to be written as a function depending on the time and radius around a line source at a constant heat flux in a plane perpendicular to the pile vertical axis in an infinite solid. This model was developed to calculate boreholes that typically have a radius of several centimeters and are very small compared to their length. This very thin borehole can therefore be considered as a line of an infinite length without any volume, which releases (absorbs) heat to (or from) the surrounding soil.

$$
G(r, t)=\frac{1}{4 \pi \lambda_{s}} \int_{r_{p}^{2} / 4 a_{s} t}^{\infty} \frac{\exp (-u)}{u} d u=\frac{1}{4 \pi \lambda_{s}} E_{1}\left(\frac{r_{p}^{2}}{4 a_{s} s}\right)
$$

For large values of the parameter $\left(a t / \mathrm{r}^{2}\right)$, where $\mathrm{a}[\mathrm{m} 2 / \mathrm{s}]$ is the thermal diffusivity, the exponential integral $\mathrm{E}_{1}$ can be simplified to:

$$
\mathrm{E}_{1}\left(\frac{\mathrm{r}_{\mathrm{p}}^{2}}{4 \mathrm{a}_{\mathrm{s}} \mathrm{t}}\right)=\ln \left(\frac{4 \mathrm{a}_{\mathrm{s}} \mathrm{t}}{\mathrm{r}_{\mathrm{p}}^{2}}\right)-\gamma \quad \frac{a_{s} t}{r_{p}^{2}} \geq 5
$$

The Infinite line source model is considered to be a simplification of the cylindrical source model (CSM) (fig. 1), that takes into account the radial dimension of the pile. The same input parameters as the infinite line source model are used. The cylindrical source model considers heat transfer outside the pile as the conduction of heat in an infinite space internally bounded by the pile wall to which a boundary condition of a constant heat flux or temperature can be applied (Ingersoll et al., 2007). The solution is described by the equation:

$$
G(r, t)=\frac{1}{4 \pi \lambda_{s}} \int_{0}^{\infty}\left(e^{-a_{s} u^{2} t}-1\right) \frac{J_{0}\left(u r_{p}\right) Y_{1}\left(u r_{p}\right)-Y_{0}\left(u r_{p}\right) J_{1}\left(u r_{p}\right)}{u^{2}\left[J_{1}^{2}\left(u r_{p}\right)+Y_{1}^{2}\left(u r_{p}\right)\right]} d u
$$

The symbols $\mathrm{J}_{0}, \mathrm{~J}_{1}, \mathrm{Y}_{0}, \mathrm{Y}_{1}$ in the equation represent the Bessel functions of the first and second kinds. For large values of the parameter (at / r2), the equation can be simplified to:

$$
G(r, t)=\frac{1}{4 \pi \lambda_{s}}\left[\ln \left(\frac{4 a_{s} t}{r_{p}^{2}}\right)-\gamma+\frac{r_{p}^{2}}{2 a_{s} t}\left(\ln \left(\frac{4 a_{s} t}{r_{p}^{2}}\right)-\gamma+1\right)\right]
$$

When the line source simplification is assumed, the formulas (4),(7) are unsuitable for small time steps, since this assumption may initially delay the temperature response on the pilot wall. With a relative error of less than $10 \%$ between the two models, they can be used in the time step (Li and Lai, 2015):

$$
t \geq \frac{5 r_{p}^{2}}{a_{s}}
$$

And with a relative error of less than $2 \%$ at the time step [3]:

$$
t \geq \frac{20 r_{p}^{2}}{a_{s}}
$$

A time step with a minimum value according to equation (10) is required when using any of these G-functions together with any of the models for $\mathrm{R}_{\mathrm{b}}$. The equations cannot be used for calculations for very long periods that approach a value equal to ( $\mathrm{Li}$ and Lai, 2015):

$$
t=\frac{H^{2}}{4 a_{s}}
$$

This limitation is due to ignoring the impact of the ground surface. This is a common disadvantage of all models that assume that soil is an infinite medium.

Another possible solution that takes into account the soil's surface temperature is the Finite line source model (FLSM) (Zeng et al., 2002). The ground is considered to be a homogeneous semi-infinite medium with an initial uniform temperature, and its thermophysical properties are constant with temperature changes. The boundary of the medium, which represents the ground surface, maintains a constant temperature throughout the calculations.

The model uses the assumptions of the line source model. Therefore, the radial dimension of the pile is ignored. The ground surface is considered to be a reflecting plane, and there is a mirror image of 


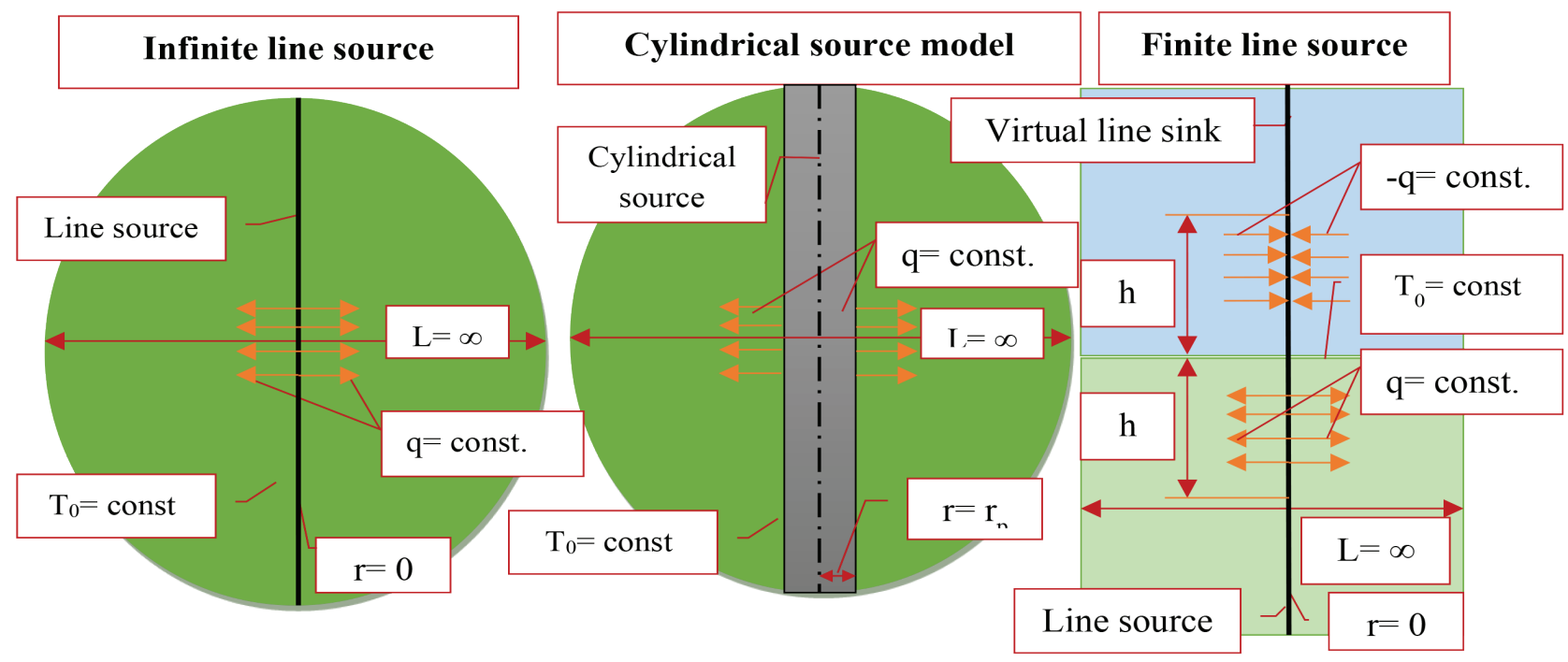

Fig. 1 Boundary conditions of the analytical models

the heat sinks. The symmetrical distribution of the line source and heat sinks can keep the ground surface temperature constant. As a representative temperature for the entire pile, the temperature, which reaches a steady state at a time approaching infinity, can be selected at the center of the pile wall. For practical applications, we define the nominal steady state to be at the time we reach a certain selected limit, in this case $98 \%$ of the steady state. Using linear regression, we can approximate the Fourrier number for this state as (Zeng et al., 2002):

$$
F O_{S}=3.8 \cdot \frac{r_{p}}{H}+0.29 \quad 0.001 \leq \frac{r_{p}}{H} \leq 0.7
$$

If the time approaches infinity, the solution to the problem can be reduced to (Zeng et al., 2002):

$$
G(r, z, t)=\frac{1}{4 \pi \lambda_{s}} \int_{0}^{H}\left\{\frac{\operatorname{erfc}\left(\frac{\sqrt{\rho^{2}+\left(z-h^{2}\right)}}{2 \sqrt{a \tau}}\right)}{\sqrt{\rho^{2}+(z-h)^{2}}}-\frac{\operatorname{erfc}\left(\frac{\sqrt{\rho^{2}+\left(z+h^{2}\right)}}{2 \sqrt{a \tau}}\right)}{\sqrt{\rho^{2}+(z+h)^{2}}}\right\} d h
$$

If the pile radius $r_{b}$ is much smaller $\left(r_{b} / H<<1\right)$ compared to its depth $\mathrm{H}[\mathrm{m}]$, the steady-state equation in the center of the well wall can be simplified to (Zeng et al., 2002):

$$
\mathrm{G}(\mathrm{r})=\frac{1}{2 \pi \lambda_{\mathrm{s}}} \ln \frac{\mathrm{H}}{\sqrt{3} \mathrm{r}_{\mathrm{b}}}
$$

Since the temperature along the pile wall significantly changes, it is not ideal to use a formula that uses the temperature in the middle of the pile wall. It is recommended to use the integral mean temperature along the pile from which we get the equation obtained by linear regression for the calculation of the G-function defined as (Zeng et al., 2002):

$$
\mathrm{G}(\mathrm{r})=\frac{1}{2 \pi \lambda_{\mathrm{s}}} \ln \frac{\mathrm{H}}{2.2 \mathrm{r}_{\mathrm{b}}}
$$

\section{EVALUATION OF THE ANALYTICAL MODELS}

The compared models were the inifinite line source model (4), cylindrical source model (6), and finite line source model (12), with the numerical model (NM). The heat transfer was modeled as a stationary and dynamic simulation. The analytical models were computed using our own calculation software, which enables the stationary and tran- sient calculation of heat flux $q[\mathrm{~W} / \mathrm{m}]$ for a pilot of any dimension, while taking into account all the above-mentioned conditions. The numerical solution was obtained by simulation using the CalA 4 software (Sikula et al., 2015). This program enables stationary and non-stationary calculations of heat conduction using a control volume method on a 2D orthogonal mesh, which can be solved as rotationally symmetrical. The verification of the rotationally symmetrical model is provided in (Sikula et al., 2014). In all the simulations, the thermophysical properties of the materials were considered to be constant, isotropic and temperature independent. The scheme of the simulated energy pile, together with the material properties and boundary conditions, can be seen in Figure 2a. A total of 100 days of pile operations were simulated during which heat was continuously extracted from the soil. The time step for the analytical models was chosen according to (3) to 96 hours. The numerical model (NM) was calculated in an hourly time step. The scheme of the geometry and boundary conditions for the NM can be seen in Figure 2b. The stationary models were calculated for the time obtained from the equation (11) using the Fourrier number, for which only the steady state heat flux was obtained (13), (14).

From the results of the dynamic simulations (Figure 3), we can see that the heat fluxes of the analytical models and their courses are very similar. The biggest differences between the analytical and numerical models occur at the end of the calculations, where they reach up to $8 \mathrm{~W} / \mathrm{m}$.

The steady-state heat fluxes deviate from the numerical solution in a range of $6-14 \%$ (Table 1). Overall, the Infinite line source model and cylindrical source model show almost identical results, with the finite line source calculations showing a higher degree of performance (about $10 \%$ ), which is caused by using more realistic boundary conditions in this case. The highest heat flux is reached by the numerical solution, but this value is higher than in reality, because it is dependent on the size of the computational domain. The state of the constant heat flux is reached sooner than in the actual operation of an energy pile.

\section{CONCLUSION}

Analytical models, which were originally designed for the calculation of boreholes, were applied to thermally-activated piles. The main disadvantages of these models (ILSM, CSM) are that they do not take into account the effect of the ground surface on the cal- 

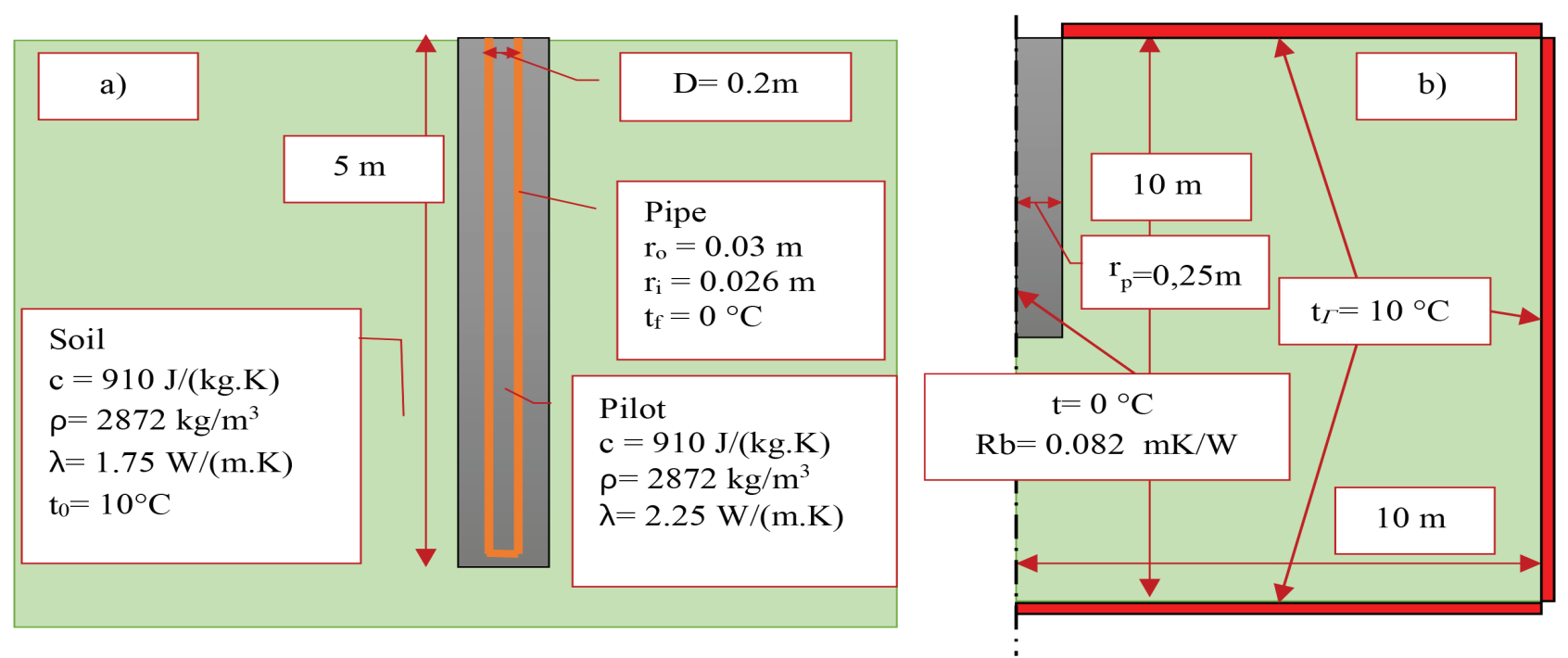

Fig. 2 a) The scheme of the modeled case, b) boundary conditions of the numerical model

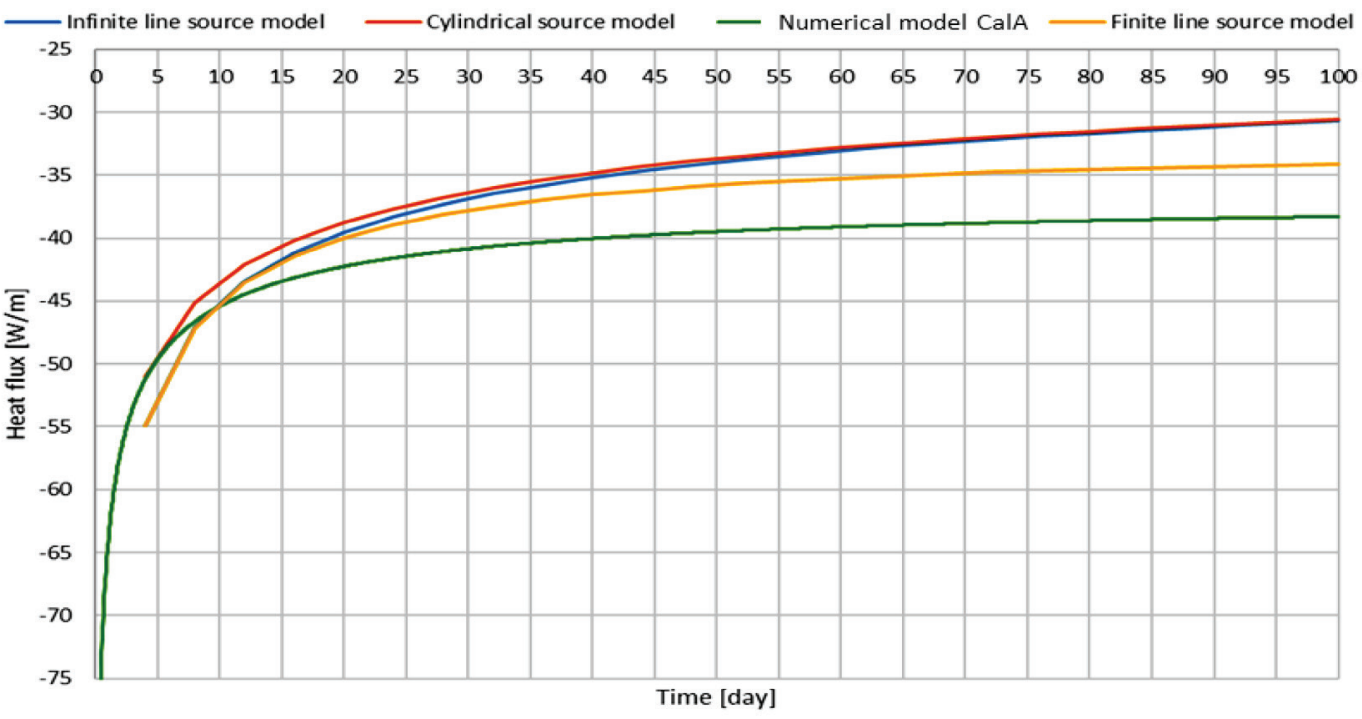

Fig. 3 Comparison of the heat fluxes of the analytical and numerical models

Tab. 1 Specific heat flow of the pile at a steady state and after 100 days

\begin{tabular}{|ccccccccc|}
\hline Time & \multicolumn{2}{c}{ steady state } & & \multicolumn{2}{c|}{ after 100 days } \\
Model & NM CalA & $\begin{array}{c}\text { FLSM - Mean } \\
\text { temperature (13) }\end{array}$ & $\begin{array}{c}\text { FLSM - Integral mean } \\
\text { temperature (14) }\end{array}$ & NM CalA & ILSM & CSM & FLSM \\
$q[\mathrm{~W} / \mathrm{m}]$ & -37.4 & -32.7 & -35.3 & -38.3 & -30.4 & -30.3 & -34.0 \\
Relative error [\%] & - & $-14 \%$ & $-6 \%$ & - & $-20.6 \%$ & $-20.9 \%$ & $-11.2 \%$ \\
\hline
\end{tabular}

culations, which is essential for energy piles, as they are generally shorter than boreholes. FLSM solves this problem in part by dividing the surface into a semi-space, but it is not possible to set a different boundary condition for the soil surface and the soil itself. Due to the different geometry of the pile compared to the borehole, these models have greater limitations to the minimum time step (8), of the order of the days. Similarly, the total calculation time (10) is shorter than for boreholes.

These analytical models are generally useful when we need to quickly determine the approximate heat output of a pile with low computational complexity. Numerical models are more suitable for physically-detailed solutions. In the future, it would be appropriate to design an analytical or hybrid model that can resolve the above-mentioned shortcomings.

\section{Acknowledgement}

This work has been funded by TAČR NCK CAMEB, Epilot Project No. TN01000056/06. 


\section{REFERENCES}

Carslaw, H. S. - Jaeger, J. C. (1959) Conduction of heat in solids. 2nd ed. Oxford: Claremore Press, 1959.

Gu, Y. - O'Neal, D. L. (1998) Development of an equivalent diameter expression for vertical U-tubes used in ground-coupled heat pumps. ASHRAE Transactions, vol. 104, Texas A\&M Univ, College Station, US: 1998, pp. 347-355.

Ingersoll, L. R. (2007) Heat Conduction - With Engineering and Geological Application. DODO Press; 2007.

Li, M. - Lai A. C. K. (2015) Review of analytical models for heat transfer by vertical ground heat exchangers (GHEs): A perspective of time and space scales. Applied Energy. 2015, 151, 178191. DOI: 10.1016/j.apenergy.2015.04.070. ISSN 03062619.
Sikula O. - Plasek J. (2015) Software CalA 4.0 (Calculation Area). version 4.0 Edu. 2015/10/05. doi:10.13140/RG.2.1.1501.7689.n̆

Šikula, O. - Mohelníková, J. - Plášek, J. (2014) Thermal analysis of light pipes for insulated flat roofs. Energy And Buildings 2014; pp. 436-444, doi:http://dx.doi.org/10.1016/j.enbuild.2014.09.044.

Zeng, H. Y. - Diao, N. R. - Fang, Z. H. (2002) A finite line-source model for boreholes in geothermal heat exchangers. Heat Transfer - Asian Research 2002, 31, 558-567. doi:10.1002/htj.10057. 